\title{
A Combustibility Study of Gaseous Pyrolysates Produced by Polyester/Cotton Blends
}

\author{
M. DAY, T. SUPRUNCHUK, and D. M. WILES
}

Textile Chemistry Section, Division of Chemistry

National Research Council of Canada

Ottawa, Ontario, Canada K1A OR6

\begin{abstract}
The thermal decomposition and flammability limits of the gaseous pyroly sates obtained from a series of polyester/cotton blends have been studied. Comparison of data obtained from intimately blended samples with control samples indicates that both physical and chemical interaction are taking place between the components during the condensed phase decomposition. Based upon the measured flammability limits and pressure rises on ignition, the importance of chemical interactions were demonstrated; however physical effects associated with heat transfer cannot be ignored. Greatest changes were observed with the $67 / 33$ polyester/cotton blends, suggesting that this may be the most difficult to flame retard.
\end{abstract}

\section{INTRODUCTION}

Clothing and textile related fires are responsible for the majority of reporm ted fire deaths in Canada and the United States. It is in an attempt to reduce the number of these fatalities that research into safer materials has been actively pursued over the last decade or so. Because polyester/cotton fibre blends combine many of the advantages associated with the individual fibres such as comfort, moisture absorption and durable press, they find widespread application in apparel and home furnishings. Unfortunately, whilst the polyester/cotton blends may have many physical and aesthetic attributes, the blends do cause problems from the flammability point of view in that it is not possible to predict the flammability behaviour of the blends from a knowledge of the burning behaviour of the individual components. One of the reasons for this lack of predictability is associated with large differences in the combustion behaviour of the components. For example, untreated cotton fabrics are relatively easy to ignite and burn, leaving a char. Polyester fabrics on the other hand are difficult to ignite because the thermoplastic material tends to shrink away from the ignition flame, and consequently, even when ignited, burn erratically with molten drips being capable of carrying the flame away from the fabric. When the two fibre types are blended together, however, the cotton is capable of forming a carbonaceous grid or "scaffold" which acts as a support for the melting

KEYWORDS

Polyester/Cotton, Flammability, Blends, Cotton/Polyester, Pyrolysates.

Issued As NRCC \#24535 
polyester enabling it to burn in much the same way as does the parafin wax at a candle's wick. In terms of the flammability behaviour of the polyester/cotton blends as measured by various test methods, although it has has been shown that blends may have oxygen-indices lower than those of the individual fibres ${ }^{1}$, the results are dependant upon moisture content ${ }^{2}$ and environmental test temperatures ${ }^{3}$. In terms of burn injury potential, however, several studies ${ }^{4-5}$ have revealed that polyester/cotton blends represent less of a hazard than $100 \%$ cotton fabrics and indicate that increased polyester content causes reductions in the burn injury potential. These complexities arise because of the interactions between the two dis similar components in the blend. Hendrix ${ }^{2}, 3$ concluded that in the burning of polyester/cotton blends, cotton was the initial fuel which the polyester augmented as the burning proceeded causing a more vigorous burning of the blend. More recent work ${ }^{7}$ however, indicates that the polyester controls the decomposition by melting and covering the cotton, thereby preventing its decomposition.

Obviously, some form of interactive behaviour is taking place during the burning of polyester/cotton blends. Miller ${ }^{8}$ indicated that these inter actions were chemical in nature and were occuring during the pyrolysis and combustion processes to produce more volatile fuels which were responsible for lower ignition times and faster burn rates than anticipated from the individual components. Meanwhile, another study ${ }^{9}$ examining ignition characteristics has indicated that the interactions are more physical in nature than chemical and the observed differences in behaviour of blended fabrics may be attributable to heat transfer effects.

In an attempt to better understand the fundamental processes taking place in these polyester/cotton blends, we decided to investigate the decomposition and flammability limits of the gaseous pyrolysates produced using a technique developed in our laboratories ${ }^{20}$. In addition to measuring the decomposition and flammability limits of intimate polyester/ cotton blends, results have also been obtained when the solid components were separated.

\section{EXPERIMENTAL}

\section{Materials}

The cotton and polyester fabrics used in this study were obtained from Test Fabrics, Inc. The cotton was $100 \%$ bleached sheeting style 405 (176 $\mathrm{g} / \mathrm{m}^{2}$ ) while the polyester was a spun woven fabric style $767\left(127 \mathrm{~g} / \mathrm{m}^{2}\right)$. The samples were prepared by passing strips of fabric through a Wiley Mill (Model 4276) at $3,500 \mathrm{rpm}$ to produce a fine fluff. The intimate blends were prepared by mixing the polyester and cotton fine fluffs together at predetermined weight ratios, blending the mixture on a ball mill followed by a pass through the Wiley Mill. Three intimate blends were produced containing $67 \%, 50 \%$ and $33 \%$ respectively of cotton.

\section{Apparatus and Test Procedure}

The experimental determinations were carried out according to the procedure used previously 10 employing the apparatus (see Fig. 1) described in detail in an earlier paper ${ }^{1}$. In the blended experiments, $200 \mathrm{mg}$ of the combined fluff is weighed out accurately into one half of the divided crum cible (e in Fig. 1) while in the separated experiments, the polyester is weighed out accurately into one half and cotton into the other half. The 


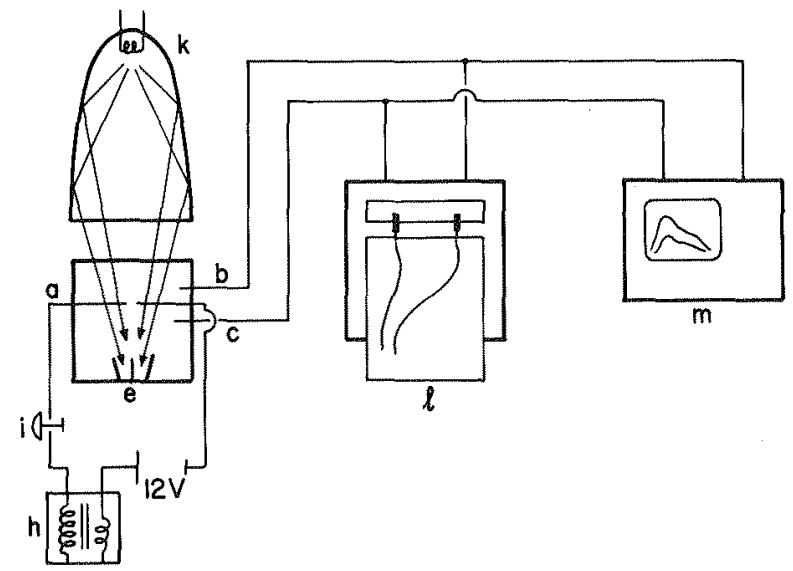

Figure 1 Schematic of the test equipment

crucible is then placed into the insulated brass combustion vessel which is $75 \mathrm{~mm}$ long and $50 \mathrm{~mm}$ in diameter. The sample is then heated in the sealed vessel by means of an external infrared heater ( $k$ ) focused on the sample through a transparent mica window. After a predetermined heating time, the gaseous pyrolysate produced is subjected to a spark discharge from the electrodes (a) generated by a power supply and ignition coil (h). The strip chart recorder (1) and storage oscilloscope (m) monitor the pressure and temperature during pyrolysis and combustion. All studies employed a lamp voltage of $80 \mathrm{~V}$ to give an incident radiative pyrolysis heat flux of about $13 \mathrm{~W} / \mathrm{cm}^{2}$. It should be pointed out that each point represents one determination with each experiment being performed on a new sample. The weight of pyrolysate produced was determined from the loss in weight of the crucible contents after each experiment. In the case of the experiments performed on the separated samples, individual weight losses were determined by cleaning and dissolving the residue in each half separately.

\section{RESULTS AND DISCUSSION}

Gases or vapours which form flammable mixtures with air or oxygen do so within specific concentration ranges defined as the flammability limits. These limits known as the "lower and upper flammability limits" are a measure of boundary limits in which a mixture of the gas or vapour with air will, if ignited, just support flame propagation. Although widely used in hazard evaluation, these values may well be dependant upon: pressure. temperature, ignition source, equipment geometry etc. In our studies, we have taken the flammability limit concept and applied it to the gaseous pyrolysates produced from decomposing polymeric materials. However, in this latter application, the flammability limits determined are also dependant upon the thermal stability of the polymeric material and the heating rate employed to generate the pyrolysate. These factors become exceedingly important in the case of polymers whose decomposition products are dependant upon the thermal conditions employed, as is the case with polyester $^{12}$ and cellulose ${ }^{13}$. For these reasons, only one set of experimental and reproducible conditions have been employed throughout this study in order that useful comparisons and interpretations can be made. 


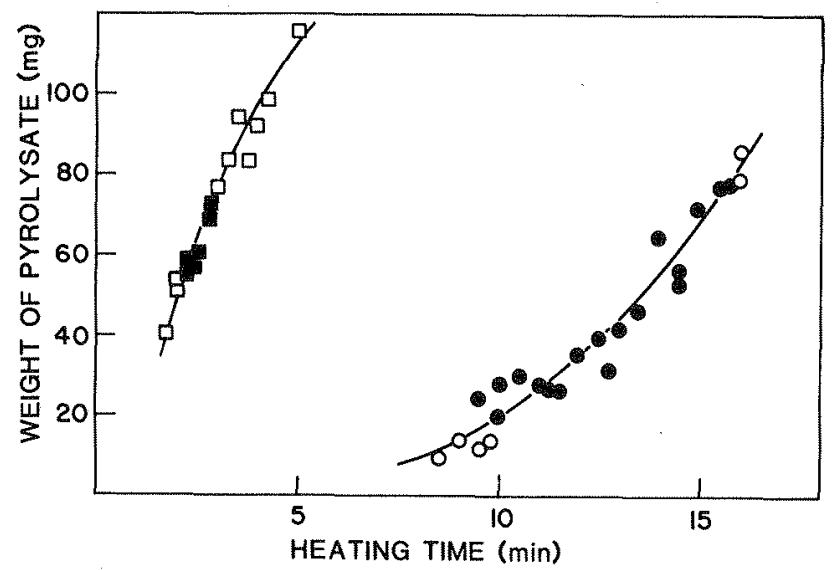

Figure 2 Weight of pyrolysate produced from cotton ( $\square$ ) and polyestex (O) with combustible mixtures denoted and

The marked difference in the thermal stability of each component is immediately evident from Figure 2 in which the weight of pyrolysate produced (weight loss of sample) is plotted as a function of heating time. This figure clearly indicates that cotton undergoes a relative rapid weight loss to produce a gaseous pyrolysate which reaches its lower flammability limit in approximately 2 minutes. However, further heating produces more pyrolysate such that the gas air mixture reaches the upper flammability limit after about 3 minutes and then enters the fuel rich zone. In contrast, the poly ester has a much slower rate of weight loss than the cotton, such that heating for a period of 9.5 minutes is required before a sufficient concentration of pyrolysate in air is attained to obtain a combustible gas air mix ture. However, in the case of the polyester, the range of gaseous pyroly sate concentrations in air capable of supporting combustion is far greater than that observed with the cotton, and it is only after heating for a period of 15.5 minutes that the upper flammability limit is reached. Thus, based upon the observed behaviour of the individual components, it would be anticipated in terms of a polyester/cotton blend, the pyrolyste from the cotton will produce a combustible gas-air mixture, which will become too rich in cotton pyrolysate products before an appreciable pyrolysate has been generated from the polyester.

The actual behaviour of the blended polyester/cotton samples are presented in Figures 3-5 for the 33/67, 50/50 and 67/33 P/C systems respectively. These graphs provide not only the data for the intimately blended samples, but also results obtained in which the corresponding weights of the individual components are placed in each side of the divided crucible. In this way it was possible to compare the data obtained from the two systems under identical experimental conditions and elucidate any possible interactions taking place as a result of the intimate blending of the two components. Examination of the data presented in Figures 3-5 clearly indicates that changes are occuring because of the mixing of the two components together. When the components are blended together, the amount of gaseous pyrolysate produced is always greater than when the comno- 


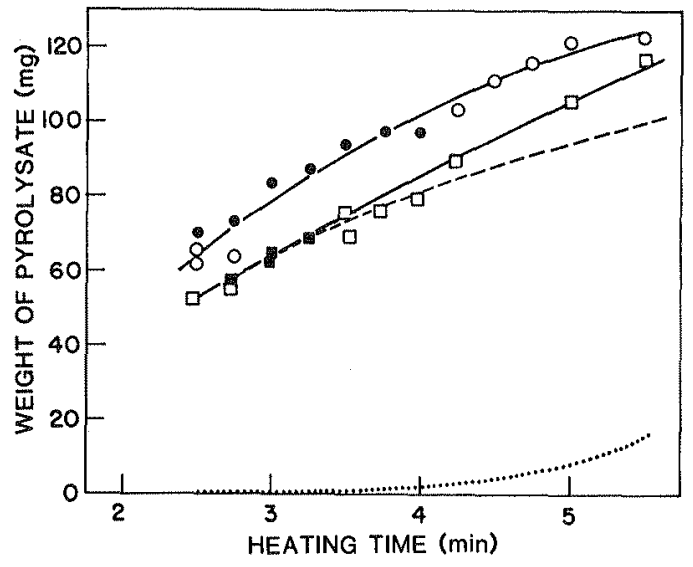

Figure 3 Total weight of pyrolysate produced from the $33 / 67$ polyester/ cotton system for blended samples ( $O$ ) and separated samples ( $\square$ ) with combustible mixtures denotes and The pyrolysate contribution of cotton and polyester to the separated systems are shown ... and ... respectively.

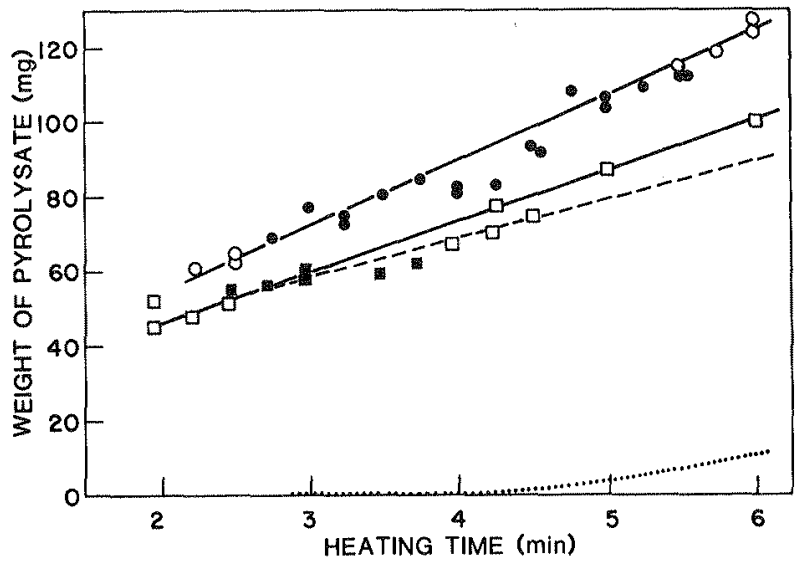

Figure 4 Total weight of pyrolysate produced from the $50 / 50$ polyester/ cotton system for blended samples ( $O$ ) and separated samples ( $\square$ ) with combustible mixtures denotes and the pyrolysate contribution of cotton and polyester to the separated systems are shown m and ... respectively.

nents are separated, with the difference appearing to increase as the amount of polyester in the system increases. This observation regarding decomposition and the production of pyrolysate is consistent with data reported in the literature ${ }^{9}$ which was explained on the basis of physical interactions based upon a heat transfer mechanism. However, Figures 3-5 also indicate that in addition to changes in the amount of pyrolysates produced, blending of the two components also results in changes in the 


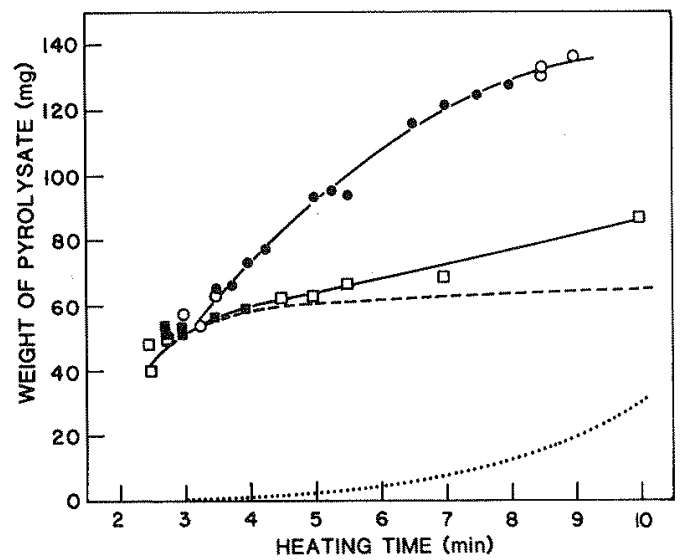

Figure 5 Total weight of pyrolysate produced from the $67 / 33$ polyester/ cotton system for blended samples ( $O$ ) and separated samples ( $\square$ ) with combustible mixtures denotes and . The pyrolysate contribution of cotton and polyester to the separated systems are shown $\ldots-m$ and ... respectively.

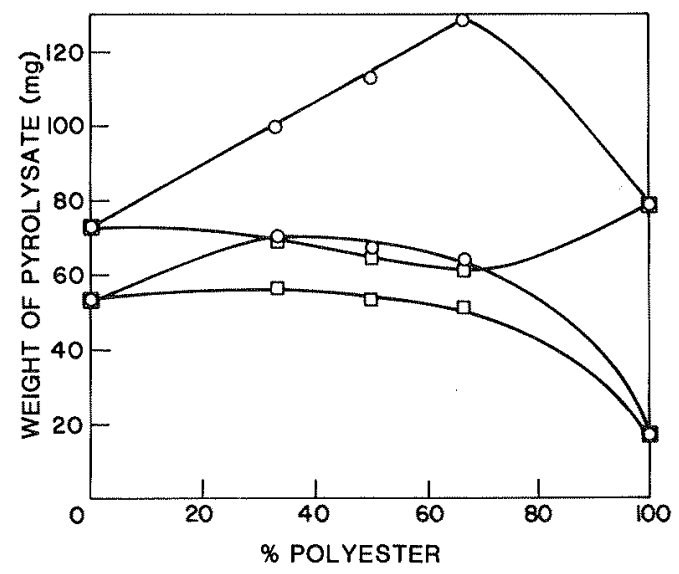

Figure 6 Flammability limits for the blended samples ( $O$ ) and separated samples ( $\square$ ) as a function of polyester in the sample.

flammability limits different to those produced with the separated system. In order to better appreciate these changes in the flammability limits as a function of composition, the data have been replotted in Figure 6 as a percentage of polyester in the systems.

Figure 6 clearly indicates that the flammability limits for the separated system are distinctly different from those obtained with the blended samples. In the case of the separated samples, the flammability limits correspond very closely to those of the pure cotton, as would be antici- 


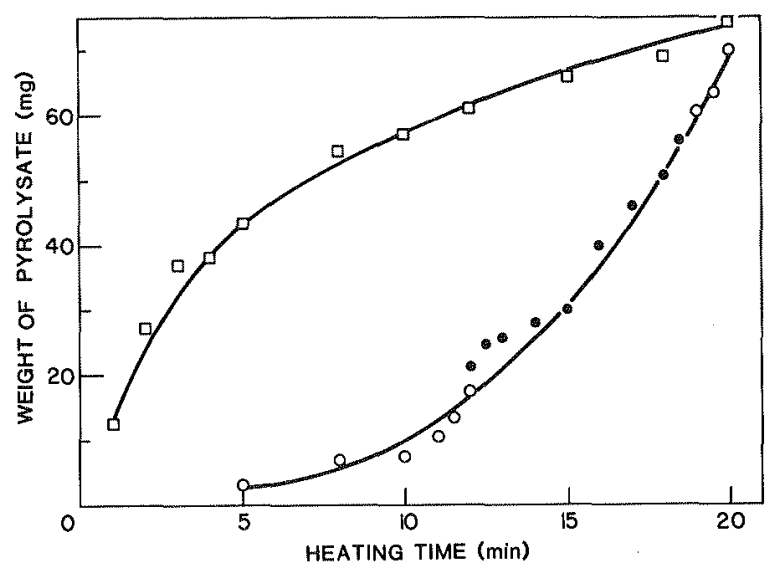

Figure 7 Total weight of pyrolysate produced from $100 \mathrm{mg}$ sample of polyester (O) and a $200 \mathrm{mg}$ of a $50 / 50$ polyester/glass fibre blend ( $\square$ ), with combustible mixture denoted

pated based upon the decomposition behaviour noted in Figure 2. Thus the limits measured for the separated systems must be associated with the pyrolysate generated from the cotton only, with no contribution from the polyester. Confirmation that the observed limits were due to the cotton were made by performing experiments with cotton only, employing sample weights of $100 \mathrm{mg}$ and $67 \mathrm{mg}$ when only slight changes in the limits were obtained comparable to those measured for the separated systems.

The observation that the blended samples have flammability limits vastly different from those obtained with the separated systems clearly indicates that the components are not decomposing independantly. In order to further elucidate the nature of the interactions additional experiments were performed with polyester blended with glass fibres to act as inert non-combustible support. The results obtained with $200 \mathrm{mg}$ samples of a $50 / 50$ polyester/glass fibre blend are given in Figure 7. Also included in this figure are the results obtained with $100 \mathrm{mg}$ sample weights of polyester. Immediately, the effect of the inert glass fibre on the decomposition of the polyester becomes evident being responsible for a marked reduction in heating time required to obtain gaseous pyrolysate (separate experiments with the glass fibres indicated no weight loss). For example after heating $100 \mathrm{mg}$ of pure polyester alone for 7 minutes only $5 \mathrm{mg}$ of pyroly sate has been produced, however, when supported by the glass fibre 50 mg has been produced in the same time period. In the case of the pure polyester, combustible pyrolysate gas/air mixtures are obtained with lower and upper flammability limits consistent with expectation. In the case of the polyester/glass blend, however, no combustible gas/air mixtures were obtained. This result is rather suprising since although it would have been anticipated that the rate of pyrolysate gas generating may increase because of physical heat transfer effects of the inert support the change in the composition of the gaseous pyrolysate such that no combustible gas mixtures were obtained was unexpected. However, in view of the large differences in the flammability limits observed with the blended polyester/cotton samples in comparison to the results with the separated system significant changes in the chemical composition of the pyrolysate 


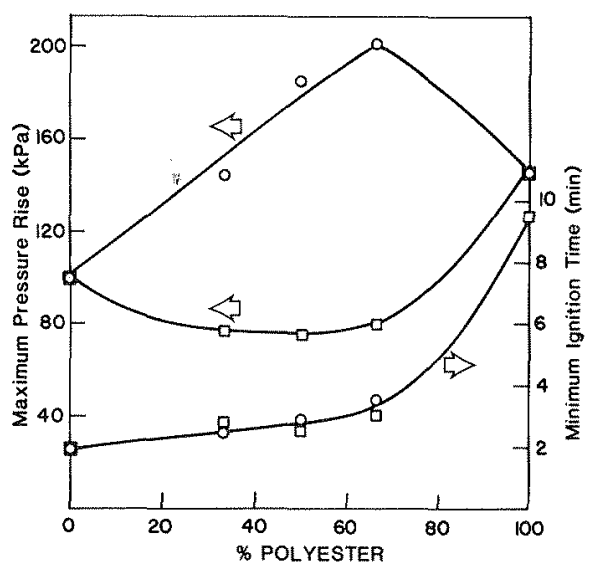

Figure 8 Minimum ignition time and maximum pressure rise on ignition for the blended samples (O) and separated samples ( $\square$ ) as a function of poly" ester in the sample.

gases must be occuring. Thus it appears that, in addition to physical interactions, chemical interactions must also be taking place during decomposition of these blends to produce a more combustible fuel than is generated in the separated system. This observation regarding the combustibility of the pyrolysate appears to confirm Miller's findings [8] which showed that the pyrolysates have a greater fuel value due to the production of larger amounts of ethylene and acetylene in polyester/cotton blends than would be predicted from the behaviour of the individual components.

The derivation of flammability limits for the gaseous pyrolysates along with the information concerning the thermal stability of the materials only represents part of the information obtainable from these combustibility studies. In terms of the decomposition rate, the time to obtain a combustible gas/aix mixture is an important parameter in any flammability evaluation in that it reflects the dynamics of the burning process. In Figure 8 the heating time to obtain a combustible pyrolysate/air mixture has been plotted as a function of polyester content. Essentially the separated and blended systems appear to behave in a similar manner, without any obvious differences. If the interactions between the two components were purely physical in nature, and associated with a heat transfer process, it would be anticipated that shorter times would have been required for the blended systems. However, if chemical interaction in addition to the physical interaction were taking place, the different chemical composition of the gaseous pyrolysates could be sufficient to explain the observed results.

When combustible pyrolysate/air mixtures were obtained in this study, the pressure increase associated with the ignition of the pyrolysate are measured and can be plotted as a function of weight of pyrolysate. In all systems studied, these plots were similar to those obtained previously ${ }^{10}$ and were characterised by Gausian shaped curves which increase sharply in regions close to the flammability limits to reach maximum values at some point close to the mid point between the limit values. For that reason, we have taken the liberty of reporting only the maximum pressure rises noted for each system studied. These results are presented in Figure 8 . 
Once again the behaviour of the polyester/cotton blended system are different to those obtained with the separated system. This observation is indicative that the chemical composition of the gaseous pyrolysate is different with the blended materials than found with the separated systems. The magnitude of the pressure rise on ignition of the pyrolysate/air mixtures may be taken as a useful indicator of the energy being released during the combustion process and is therefore capable of providing information on the potential heat feedback during the burning process. In the case of the separated systems, the energy released during ignition of the pyroly sate closely resembles that of the cotton alone, a fact to be expected based upon the data obtained which indicate that the cotton is the source of the pyrolysate. In the case of the polyester/cotton blends, the energy release as measured by the maximum pressure rise on ignition lie above the line connecting the values for the individual components with two of the blends having values greater than that for the polyester alone. These data while comparable to those obtained by Miller [8] appear to be in conflict with those reported by Yeh [14] who obtained a "minimum" heat release for a $45 / 55$ polyester/cotton blend based upon calorimetric studies. It should be pointed out, however, that in our study, the ignition and combustion are occuring in a closed reaction vessel which by its nature limits the amount of air available for combustion. Although the pyrolysate/air mixtures are within the flammability limits in order to obtain ignition, the consumption of air during combustion undoubtedly will quickly result in oxygen starvation. In the previously reported studies $[8,14]$, the fabrics are being burnt in systems in which the oxygen starvation may not be a problem. although Miller's data do show some dependance upon air flow rate. How ever, irrespective of the cause of these discrepancies, the results are indicative of interactions between the two components.

\section{CONCLUSIONS}

The thermal decomposition of intimate blends of polyester/cotton is a complex process in which interactions take place in the condensed phase prior to the production of the gaseous pyrolysate which serves as the fuel for the combustion process. Because of the large changes in the flammability limits of the blends compared to the limits for the pure polyester and cotton, the chemical composition of the gaseous pyrolysate fuel must be significantly different. Based upon the results of this study, it appears that both physical and chemical interactions are occuring which can account for the changes in the chemical composition of the gaseous fuel being produced when these blends are heated during thermal decomposition leading to combustion. In view of this evidence of substantial chemical interac tions in the blends, the difficulty in obtaining successful flame retardant systems for the polyester/cotton blends (based upon existing technology for the individual components) is understandable.

\section{REFERENCES}

1. Tesoro, G.C. and Meiser, C.H.: "Some Effects of Chemical Composition on the Flammability Behaviour of Textiles", Text. Res. J., 40 430-436, (1970).

2. Hendrix, J.E., Drake, G.L. and Reeves, W.A.: "Effects of Fabric Weight and Construction on Oxygen Index (OI) Values of Cotton Cellulose", J. Fire and Flammability, $\underline{3}, 38-45$ (1972).

3. Hendrix, J.E., Drake, G.L. and Reeves, W.A.: "Effects of Temperature on Oxygen Index Values", Text, Res. J., 41, 360 (1971). 
4. Bercaw, J.R., Jordan, K.G. and Moss, A.Z.: "Estimating Injury from Burning Garments and Development of Concepts for Flammability Tests". Fire Standards and Safety, ASTM STP 614, Ed., Robertson, A.F.: American Society for Testing and Materials, p. 55-90 (1977).

5. Langstaff, W.I. and Trent, L.C.: "The Effect of Polyester Fibre Content on the Burn Injury Potential of Polyester/Cotton Blend Fabrics", J. Consum. Prod. Flam. 7 (1) 26-39 (1980).

6. Umbach, K.H.: "Comparative Studies of The Burning Behaviour of Textiles from Polyester/Cotton and Pure Cotton", Fire Mater., 5 (1) 24-32 (1981).

7. Barker, R.H. and Drews, M.J.: "Development of Flame Retardants for Polyester/Cotton Blends", NBS-GCR-ETIP 76-22, National Bureau of Standards, Washington, D.C. (Sept. 1976).

8. Miller, B., Martin, J.R., Meiser, C.H. and Gorgiallo, M.: "The

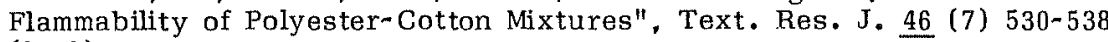
(1976).

9. Pintauro, E.M. and Buchanan, D.R.: "Ignition Process in Single and Multicomponent Polyester/Cotton Textile Structures", Text. Res. J. $\underline{49}$ (6) 326-334 (1979).

10. Day, M., Suprunchuk, T., and Wiles, D.M.: " Flammability Limits of Some Polymer Pyrolysate-Air Mixtures", J. Appl. Polym. Sci. , 28, 449-460 (1983).

11. Day, M., Suprunchuk, T. and Wiles, D.M.: "Combustion and Pyrolysis of Poly (ethylene terephthalate). I. The Role of Flame Retardants on Products of Pyrolysis", J. Appl. Polym. Sci., 26 3085-3098 (1981).

12. Day, M., Wiles, D.M.: "Influence of Temperature and Environment on the Thermal Decomposition of Poly (ethylene terepthalate) fibres with and without the Flame Retardant Tris (2,3-dibromopropyl) Phosphate". J. Anal. Appl. Pyrol. 7 65-82 (1984).

13. Shafizadeh, F, Farneaux, R.H., Cochran, T.G., Scholl, J.P. and Sakai, Y.: "Production of Levoglucosan and Glucose from Pyrolysis of Cellulosic Materials", J. Appl. Polym. Sci., 23 3525-3539 (1979).

14. Yeh, K., Valente, J.A.: "Calorimetric Study of Polyester/Cotton Blend Fabrics I. Combustion and Effects of Partial Retardants", J. Fire Ret. Chem. 6 92-124 (1979). 